\title{
On The Linear Behaviour of the Throughput of IEEE 802.11 DCF in Non-Saturated Conditions
}

\author{
F. Daneshgaran, M. Laddomada, F. Mesiti, and M. Mondin
}

\begin{abstract}
We propose a linear model of the throughput of the IEEE 802.11 Distributed Coordination Function (DCF) protocol at the data link layer in non-saturated traffic conditions. We show that the throughput is a linear function of the packet arrival rate (PAR) $\lambda$ with a slope depending on both the number of contending stations and the average payload length. We also derive the interval of validity of the proposed model by showing the presence of a critical $\lambda$, above which the station begins operating in saturated traffic conditions.

The analysis is based on the multi-dimensional Markovian state transition model proposed by Liaw et al. with the aim of describing the behaviour of the MAC layer in unsaturated traffic conditions. Simulation results closely match the theoretical derivations, confirming the effectiveness of the proposed linear model.
\end{abstract}

Index Terms-DCF, Distributed Coordination Function, IEEE 802.11, MAC, saturation, throughput, unsaturated.

\section{INTRODUCTION}

Modelling of the DCF at the MAC layer of the series of IEEE 802.11 standards has recently garnered interest in the scientific community [1]-[6]. After the seminal work by Bianchi [1] who proposed a bi-dimensional Markov model of the back-off stage procedure adopted by the DCF in saturated conditions, many papers have focused on various facets of basic access mechanism providing extensions to most recent versions of the IEEE 802.11 series of standards [7]. Recently, in [3] the authors proposed a novel fixed-point analysis of the DCF providing an effective framework for analyzing single cell IEEE 802.11 WLANs without resorting to the bidimensional contention model [1].

Practical networks usually operate in non-saturated conditions and data traffic is mainly bursty. Under these operating conditions, Bianchi's model does not describe accurately the behaviour of the throughput at the MAC layer. In this respect, in [4]-[5] the authors proposed two different bi-dimensional Markov models accounting for unsaturated traffic conditions, extending the basic bi-dimensional model proposed in [1].

In this paper we take a different approach with respect to works [4]-[5]. Upon starting from the bi-dimensional model proposed by Liaw et al. in [4], we show that the behaviour of the throughput of the IEEE 802.11 DCF in unsaturated conditions can be described by a linear relation that, with respect to the PAR $\lambda$, depends on two network parameters: the number $N$ of contending stations, and the average size $E[P L]$ of the transmitted packets. This is one of the key

F. Daneshgaran is with ECE Dept., CSU, Los Angeles, USA.

M. Laddomada, F. Mesiti, and M. Mondin are with DELEN, Politecnico di Torino, Italy. contribution of the paper: no simulations are needed for throughput evaluation since it can be theoretically predicted employing the model $S(\lambda)=N \cdot E[P L] \lambda$ developed in Section III. Of course, the limit of validity of such a model has to be clearly identified, and it represents another contribution of this paper. To this end, we derive the interval of validity of the proposed model with respect to the PARs at the MAC layer. We demonstrate the existence of a critical PAR, $\lambda_{c}$, which discriminates the unsaturated region, characterized by the range $\lambda \in\left[0, \lambda_{c}\right)$, from the saturation zone identified by any $\lambda \in\left[\lambda_{c},+\infty\right)$.

For conciseness, we invite the interested reader to refer to [4] for many details on the considered bi-dimensional Markov model, and references therein to get a picture of the topic addressed in this letter. Briefly, Liaw et al. extended the saturated Bianchi's model by introducing a new idle state, not present in the original Bianchi's model, accounting for the case in which the station buffer is empty, after a successful completion of a packet transmission. The main advantages of such a model rely on its simplicity and the effectiveness in describing the dynamics of the DCF in unsaturated traffic conditions, while basic hypotheses are the same as in Bianchi's model.

Paper outline is as follows. In section II, we briefly recall the main probabilities needed for developing the proposed linear model, evaluate the throughput and present the adopted traffic model. Finally, Section III presents the linear model of the throughput along with simulation results.

\section{Problem formulation And TrafFic MOdel}

The bi-dimensional contention Markov model proposed in [4] governs the behaviour of each contending station through a series of states indexed by the pair $(i, k), \forall i \in$ $[0, m], k \in\left[0, W_{i}-1\right]$, whereby $i$ identifies the backoff stage, and $k \in\left[0, W_{i}-1\right]$ the backoff counter. The other parameters needed in the proposed framework can be summarized as follows: $\tau$ is the probability that a station starts a transmission in a randomly chosen slot time (ST), $q$ is the probability that there is at least a packet in the queue after a successful transmission, $W_{i}=2^{i} W_{0}, \forall i \in[1, m]$, is the size of the $i$ th contention window, $W_{0}$ is the minimum size of the contention window, $P_{I, 0}$ is the probability of having at least one packet to be transmitted in the queue when the system is in idle state, and $p$ is the collision probability defined as in [1]

$$
p=1-(1-\tau)^{N-1}
$$

Stationary probability $b_{I}$ of being in the idle state is:

$$
b_{I}=(1-q) b_{0,0} / P_{I, 0}
$$


whereby $b_{0,0}$ is defined as follows:

$$
b_{0,0}=\frac{1-b_{I}}{\alpha}, \alpha=\frac{1}{2}\left[\frac{1-(2 p)^{m+1}}{1-2 p} W_{0}+\frac{1-p^{m+1}}{1-p}\right]
$$

By employing the normalization condition [1], it is possible to obtain:

$$
\tau=\sum_{i=0}^{m} b_{i, 0}=\sum_{i=0}^{m} p^{i} \cdot b_{0,0}=\varepsilon \cdot b_{0,0}, \varepsilon=\frac{1-p^{m+1}}{1-p}
$$

Next line of pursuit is the computation of the system throughput. Putting together Eq.s (1), (4), a nonlinear system can be defined and solved numerically, obtaining the values of $\tau$ and $p$. The solution of the previous system is used for evaluating the throughput, defined as the ratio between the average payload information transmitted in a ST and the average length, $T_{a v}$, of a ST:

$$
S=P_{t} \cdot P_{s} \cdot E[P L] / T_{a v}
$$

whereby $E[P L]$ is the average packet payload length (expressed in bits), $P_{t}$ is the probability that there is at least one transmission in the considered ST, with $N$ stations contending for the channel, each transmitting with probability $\tau$, i.e., $P_{t}=$ $1-(1-\tau)^{N}$. Probability $P_{s}$ is the conditional probability that a packet transmission occurring on the channel is successful:

$$
P_{s}=N \tau(1-\tau)^{N-1} / P_{t}
$$

Upon noting that, in a given ST, a station can reside in one of three possible kind of states, namely the idle state $I$ where the station spends $T_{I}$, the backoff states where the station spends $T_{B O}$, and the transmitting states in which the station spends $T_{T X}$, then the average duration $T_{a v}$ of a ST easily follows

$$
\begin{aligned}
T_{a v} & =b_{I} \cdot T_{I}+\tau \cdot T_{T X}+\sum_{i=0}^{m} \sum_{k=1}^{W_{i}-1} b_{i, k} \cdot T_{B O} \\
& =b_{I} \cdot T_{I}+\left[\varepsilon \cdot T_{T X}+\theta \cdot T_{B O}\right] \cdot b_{0,0}
\end{aligned}
$$

where $\varepsilon$ is defined in (4), and

$$
\theta=\frac{1}{2}\left[\frac{1-(2 p)^{m+1}}{1-2 p} W_{0}-\frac{1-p^{m+1}}{1-p}\right]
$$

Let us define the time durations $T_{T X}, T_{B O}$ and $T_{I}$ in (7). Transmission time $T_{T X}$ can be evaluated by noting that a station can experience two possible events: it successfully transmits over the channel or it encounters a collision. By doing so, $T_{T X}$ can be defined as follows:

$$
T_{T X}=(1-p) \cdot T_{s}+p \cdot T_{c}
$$

whereby $T_{c}$ and $T_{s}$ are, respectively, the average time a channel is sensed busy due to a collision, and the successful data frame transmission time [1], [4].

Backoff time duration $T_{B O}$ can be evaluated by considering the following two possibilities. A station can reside in a backoff slot of duration $\sigma$ if no other station is transmitting in the same ST, or for a time $T_{T X}$ due to a collision with at least another station occupying the channel:

$$
T_{B O}=\left(1-P_{t x[N-1]}\right) \cdot \sigma+P_{t x[N-1]} \cdot T_{T X}
$$

TABLE I

NUMERICAL RESULTS.

\begin{tabular}{l||c|c|c}
\hline \hline$N$ & 10 & 20 & 30 \\
\hline$S_{m}[\mathrm{Mbps}]$ & 9.118 & 8.73 & 8.608 \\
\hline$\lambda_{c}[\mathrm{pkt} / \mathrm{s}]$ & 111.2 & 53.235 & 34.99 \\
\hline \hline
\end{tabular}

whereby $P_{t x[N-1]}=1-(1-\tau)^{N-1}$ corresponds to the probability that at least a station, other than the tagged one, is transmitting in a ST.

For the sake of defining the traffic model employed for performance verification, we need to define both the access time $T_{A}$ (this is the average time a station spends through the various backoff stages before transmitting a packet) and the service time $T_{S}$. From [4], $T_{A}$ can be defined as follows:

$$
T_{A}=\frac{\sum_{i=0}^{m} p^{i} \cdot \frac{W_{i}}{2} \cdot T_{B O}}{\sum_{i=0}^{m} p^{i}}=\frac{W_{0}}{2 \varepsilon} \frac{1-(2 p)^{m+1}}{1-(2 p)} \cdot T_{B O}
$$

whereby $\varepsilon$ is as defined in (4). On the other hand, $T_{S}$ [6], i.e., the time elapsed from the moment a packet is taken from the queue to the instant in which it is successfully transmitted, can be defined as $T_{S}=T_{A}+T_{T X}$.

As far as $T_{I}$ is concerned, we resort to the definition [4]:

$$
T_{I}=\frac{\left[\varepsilon \cdot T_{T X}+\theta \cdot T_{B O}\right] \cdot b_{0,0}}{1-b_{I}}=\frac{\left[\varepsilon \cdot T_{T X}+\theta \cdot T_{B O}\right]}{\alpha}
$$

where last equality stems from (3).

The employed traffic model is $M / G / 1 / K$. Probabilities $q$ and $P_{I, 0}$ in our model can be defined as follows:

$$
q=1-\pi_{0}=1-\frac{1-\rho}{1-\rho^{K+1}}, \quad P_{I, 0}=1-e^{-\lambda \cdot T_{I}}
$$

where $\pi_{0}$ is the probability of an empty system [8], $\rho=\lambda \cdot T_{S}$, $q$ follows from M/G/1/K queuing theory [8], while $P_{I 0}$ stems from the fact that for exponentially distributed interarrival times with mean $1 / \lambda$ the probability of having at least one packet arrival during time $T$ is equal to $1-e^{-\lambda \cdot T}$.

Employing (2), for a $K-1$-length queue, we have:

$$
b_{I}=\frac{1-\rho}{1-\rho^{K+1}} \cdot \frac{1}{1-e^{-\lambda \cdot T_{I}}} \cdot b_{0,0}
$$

\section{THE LINEAR MODEL AND SIMULATION RESULTS}

A model of the throughput in non-saturated traffic conditions, along with its dependence on some key network parameters, can be derived by analyzing (5) in the limit $\lambda \rightarrow 0$.

Let us write the throughput in (5) as a function of $\tau$. By employing (6), the numerator can be rewritten as $P_{t} P_{s} E[P L]=$ $N \tau(1-\tau)^{N-1} E[P L]$. As far as the denominator is concerned, upon substituting (12) in (7), and remembering that $b_{0,0}=$ $\left(1-b_{I}\right) / \alpha$, after some algebra, it is possible to obtain the following relation $T_{a v}=\left[\varepsilon \cdot T_{T X}+\theta \cdot T_{B O}\right] / \alpha$. By collecting the previous relations, the throughput can be rewritten as:

$$
S(\tau)=\frac{N \tau(1-\tau)^{N-1} E[P L] \alpha(\tau)}{\varepsilon(\tau) \cdot T_{T X}(\tau)+\theta(\tau) \cdot T_{B O}(\tau)}
$$

whereby we highlighted the dependence on $\tau$ of the terms $\alpha, \varepsilon, T_{T X}, \theta$, and $T_{B O}$. Upon noting that (3) yields $\lim _{\lambda \rightarrow 0} b_{0,0}=0$, from (4) it follows $\tau \rightarrow 0$ as well. In the limit $\tau \rightarrow 0$, it is straightforward to demonstrate the following 
TABLE II

TYPICAL NETWORK PARAMETERS

\begin{tabular}{c|c||c|c}
\hline \hline MAC header & 28 bytes & Propag. delay $\tau_{p}$ & $1 \mu \mathrm{s}$ \\
\hline PLCP Preamble & 144 bit & PLCP Header & 48 bit \\
\hline PHY header & 24 bytes & Slot time & $20 \mu \mathrm{s}$ \\
\hline PLCP rate & $1 \mathrm{Mbps}$ & $\mathrm{W}_{0}$ & 32 \\
\hline No. back-off stages, $\mathrm{m}$ & 5 & $\mathrm{~W}_{\max }$ & 1024 \\
\hline Payload size & 1025 bytes & SIFS & $10 \mu \mathrm{s}$ \\
\hline ACK & 14 bytes & DIFS & $50 \mu \mathrm{s}$ \\
\hline ACK timeout & $364 \mu \mathrm{s}$ & EIFS & $364 \mu \mathrm{s}$ \\
\hline \hline
\end{tabular}

relations: $p \rightarrow 0$ (from (11)), $\varepsilon(\tau) \rightarrow 1$ (from (4)), $\theta(\tau) \rightarrow$ $\frac{W_{0}-1}{2}$ (from (8)), $\alpha(\tau) \rightarrow \frac{W_{0}+1}{2}$ (from (3)), $T_{T X}(\tau) \rightarrow T_{s}$ (from (9)), and $T_{B O}(\tau) \rightarrow \sigma$ (from (10)).

Upon substituting the derivations above in (15), the limit $\lim _{\tau \rightarrow 0} S(\tau)=0=\left[\left.S(\tau)\right|_{\tau=0}\right]$ easily follows.

Upon employing the Taylor's formula around $\tau \approx 0$, the throughput $S(\tau)$ can be well approximated as follows:

$$
S(\tau) \approx\left[\left.S(\tau)\right|_{\tau=0}\right]+\left[\left.\frac{\partial S(\tau)}{\partial \tau}\right|_{\tau=0}\right] \tau=\left[\left.\frac{\partial S(\tau)}{\partial \tau}\right|_{\tau=0}\right] \tau
$$

As far as $\tau \approx 0,(1-\tau)^{N-1} \approx 1-(N-1) \tau+o(\tau)$; therefore, the approximation $N \tau(1-\tau)^{N-1} \approx N \tau+o(\tau)$ holds as well. As a consequence, (15) can be rewritten as follows:

$$
S(\tau) \approx \frac{N \cdot E[P L] \cdot \frac{W_{0}+1}{2}}{T_{s}+\frac{W_{0}-1}{2} \cdot \sigma} \cdot \tau
$$

Next line of pursuit consists in expressing $\tau$ in terms of the PAR $\lambda$. By using the MacLaurin expansion of the exponential $e^{-\lambda T_{I}} \approx 1-\lambda T_{I}+o(\lambda)$, from (14) it is $b_{0,0} \approx \lambda T_{I}$ as $\lambda \rightarrow$ 0 . On the other hand, Equ. (12) yields $\lim _{\lambda \rightarrow 0} T_{I}=\left[T_{s}+\right.$ $\left.\frac{W_{0}-1}{2} \sigma\right] / \frac{W_{0}+1}{2}$. Finally, in the limit $\lambda \rightarrow 0$, Equ. (4) yields $\tau \rightarrow b_{0,0} \approx \lambda \cdot\left[T_{s}+\frac{W_{0}-1}{2} \sigma\right] / \frac{W_{0}+1}{2}$. Upon substituting the previous mathematical derivations in (16), the throughput can be approximated as follows:

$$
S(\lambda) \simeq N \cdot E[P L] \cdot \lambda
$$

It is interesting to estimate the interval of validity $\left[0, \lambda_{c}\right]$ of the linear throughput model proposed in (17). An appropriate value of $\lambda_{c}$ can be obtained by finding the abscissa $\lambda$ corresponding to the intersection of the straight line (17) with the horizontal line passing through the maximum $S_{m}$ of the throughput. To this end, after expressing any term involved in (5) as a function of $\tau$, the maximum throughput $S_{m}$ can be obtained in two steps. First, one has to find the value $\tau_{m}$ for which the throughput gets maximized. This can be easily obtained by equating to zero the derivative of (5) with respect to $\tau$, and then solving for $\tau$. In the second step, the maximizing value $\tau_{m}$ can be substituted into $S(\tau)$ for obtaining $S_{m}=S(\tau)_{\tau_{m}}$. Finally, $\lambda_{c}$ is the value of $\lambda$ for which the following holds:

$$
\lambda_{c}=\lambda: N \cdot E[P L] \cdot \lambda=S_{m}
$$

Table [] shows the values of $\lambda_{c}$, found numerically, along with the respective values of $S_{m}$, for various values of $N$. We considered the bit rate $54 \mathrm{Mbps}$ for the protocol IEEE 802.11g. The linear model (17) shows a close agreement with both theoretical (continuous curves) and simulated throughput

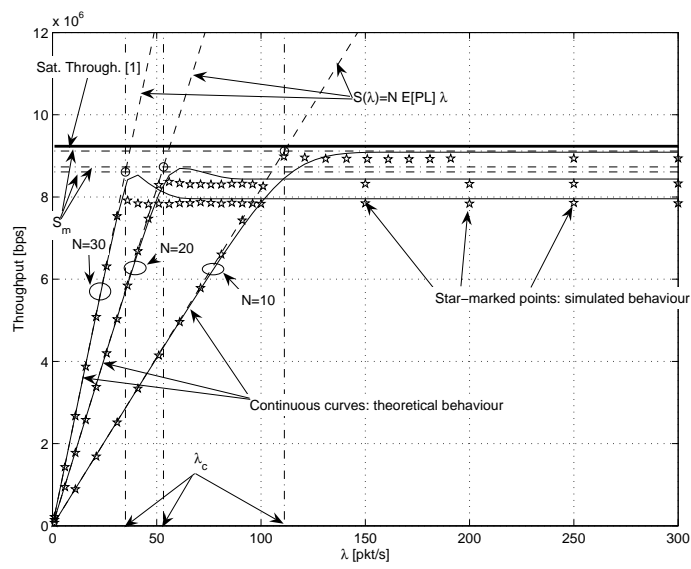

Fig. 1. Throughput for the 2-way mechanism as a function of the PAR $\lambda$, for three different number $N$ of contending stations. Straight dashed lines refer to the linear model of the throughput in 17 .

curves. Fig. 11 shows the straight lines in (17) (dashed lines) for three different values of $N$. The figure also shows the values of $S_{m}$ (horizontal dash-dot lines) along with the three values of $\lambda_{c}$ deduced from (18) and noted in Table \. Simulated values (star-marked points) have been obtained with ns-2 by using settings noted in Table II along with standard 54Mbps 802.11 parameterizations [7], which are also the standard parameters defined in ns-2. Within ns-2, $N$ stations have been randomly placed in a square area with edge size equal to $50 \mathrm{~m}$ using a uniform distribution. All simulation results in Fig. 1 are obtained with a 95\% confidence interval lower than 15kbps.

Fig. 11 shows that the maximum achievable throughput does not exceed 10Mbps (@N=10) despite the maximum bit rate employed (54Mbps): this throughput penalty is essentially due to the fact that the control packet and the PLCL header are transmitted at $1 \mathrm{Mbps}$ no matter the operating transmission mode. As a reference throughput performance, we show the maximum saturation throughput (labelled "Sat. Through. [1]" in Fig. 11 found in [1] in case the $N$ contending stations transmit at the optimal transmission probability $\tau$. Notice that, throughput penalty with respect to $54 \mathrm{Mbps}$ is well predicted by the theoretical formulation presented in [1].

\section{REFERENCES}

[1] G. Bianchi, "Performance analysis of the IEEE 802.11 distributed coordination function", IEEE JSAC, Vol.18, No.3, March 2000.

[2] G. Bianchi and I. Tinnirello, "Remarks on IEEE 802.11 DCF performance analysis", IEEE Comm. Letters, Vol.9, No.8, Aug. 2005.

[3] A. Kumar, E. Altman, D. Miorandi, and M. Goyal, "New insights from a fixed-point analysis of single cell IEEE 802.11 WLANs", to appear on IEEE/ACM Trans. on Networking, 2007, now available on IEEExplorer.

[4] Y.S. Liaw, A. Dadej, and A.Jayasuriya, "Performance analysis of IEEE 802.11 DCF under limited load", In Proc. of Asia-Pacific Conference on Communications, Vol.1, pp.759 - 763, 03-05 Oct. 2005.

[5] D. Malone, K. Duffy, and D.J. Leith, "Modeling the 802.11 distributed coordination function in non-saturated heterogeneous conditions", IEEE ACM Trans. on Networking, vol. 15, No. 1, pp. 159172, Feb. 2007.

[6] W. Lee, C. Wang, and K. Sohraby, "On use of traditional M/G/1 model for IEEE 802.11 DCF in unsaturated traffic conditions", In Proc. of IEEE WCNC, 2006, pp. 19331937.

[7] IEEE Standard for Wireless LAN Medium Access Control (MAC) and Physical Layer (PHY) Specifications, November 1997, P802.11

[8] G. Bolch, S. Greiner, H. de Meer, and K.S. Trivedi, Queueing Networks and Markov Chains, Wiley-Interscience, 2nd edition, 2006. 